TRANSACTIONS OF THE

AMERICAN MATHEMATICAL SOCIETY

Volume 350, Number 5, May 1998, Pages 1957-1975

S 0002-9947(98)01925-4

\title{
GENERALIZED HESTENES' LEMMA AND EXTENSION OF FUNCTIONS
}

\author{
MASSIMO FERRAROTTI AND LESLIE C. WILSON
}

\begin{abstract}
Suppose we have an $m$-jet field on $V \subset \mathbf{R}^{n}$ which is a Whitney field on the nonsingular part $M$ of $V$. We show that, under certain hypotheses about the relationship between geodesic and euclidean distance on $V$, if the field is flat enough at the singular part $S$, then it is a Whitney field on $V$ (the order of flatness required depends on the coefficients in the hypotheses). These hypotheses are satisfied when $V$ is subanalytic. In Section II, we show that a $C^{2}$ function $f$ on $M$ can be extended to one on $V$ if the differential $d f$ goes to 0 faster than the order of divergence of the principal curvatures of $M$ and if the first covariant derivative of $d f$ is sufficiently flat. For the general case of $C^{m}$ functions with $m>2$, we give a similar result for codim $M=1$ in Section III.
\end{abstract}

\section{INTRODUCTION}

Since H. Whitney proved his important extension theorem, introducing the notion of "Whitney field" ([Wh]; classical references are $[\mathrm{M}]$ and $[\mathrm{T}]$ ), various papers have been dedicated to the study of this subject: we recall among the others $[\mathrm{Bi}]$, $[\mathrm{H}],[\mathrm{S}]$ and the very recent $[\mathrm{KP}]$. Whitney fields are the restrictions to $V$ of jets of smooth functions on $\mathbf{R}^{n}$. If $V$ is a smooth submanifold, each smooth function on $V$ has many consistent Whitney fields on $V$. A natural question from the viewpoint of singularity theory arises when $V$ is singular. In this case we may consider functions which are smooth on the nonsingular part and just continuous all over $V$, and ask for conditions of extendibility. In particular, we may look for conditions of "flatness" at the singular locus of $V$. This paper is an attempt in this direction.

In Section I we first give a generalization of Hestenes' Lemma, which we call Generalized Hestenes' Lemma (for the classical Hestenes' Lemma, see Lemma 1 of $[\mathrm{H}]$ or $[\mathrm{T}]$, p. 80$)$. More precisely, if we have an $m$-jet field on $V$ which is a Whitney field on the nonsingular part $M$ of $V$ and which is "flat" enough at the singular part $S$, then it is a Whitney field on $V$. In order that this result holds, we need to assume that $V$ fulfils some hypotheses given as a comparison between geodesic and euclidean distance on $V$. We point out that we get extension in the same differentiability class while other known results of this type involve a loss of differentiability (compare e.g. with Proposition 3.4 of [BiMP]). Next we show in Proposition I-1 that the hypotheses of this Generalized Hestenes' Lemma are

Received by the editors January 24, 1996 and, in revised form, August 12, 1996.

1991 Mathematics Subject Classification. Primary 58C20; Secondary 53C40.

Key words and phrases. Whitney fields, singularities.

The first author was partially supported by GNSAGA (CNR), MURST. This work was partially supported by Eurocontract CHRX-CT94-0506. 
satisfied when $V$ is assumed to be subanalytic. At the end of Section I, we add some specific considerations about the isolated singularity case.

Section II is devoted to the application of our previous work to the extension of $C^{2}$ functions. We obtain in Theorem II-1 that a $C^{2}$ function $f$ on $M$ can be extended to one on $V$ if the differential $d f$ goes to 0 faster than the order of divergence of the principal curvatures of $M$ and if the first covariant derivative of $d f$ is sufficiently flat. These conditions arise from the choice of the orthogonal tubular neighborhood of $M$ to extend $f$ locally.

For the general case of $C^{m}$ functions with $m>2$, we give a result for codim $M=$ 1 in Section III. Here some work has to be done to give an estimate on the higher order derivatives (Theorem III-1) and the resulting flatness conditions on $f$ are far from being as nice as in Section II (see Definition III-2); nevertheless they still appear meaningful and they allow us to generalize in a slightly stronger way the extension result of Section II to any finite order of differentiability when we are in codimension 1 (Theorem III-2). One line of further inquiry will certainly be to search for simpler and less restrictive hypotheses under which the extension property holds.

\section{A generalization of Hestenes' Lemma}

We recall the terminology and notation of [T], Chapter IV. Let $k \in \overline{\mathbf{N}}^{n}, \overline{\mathbf{N}}=$ $\mathbf{N} \cup\{0\}$, and let $F=\left(F^{k}\right)_{|k| \leq m}$ be an $m$-Taylor field (or $m$-jet field) on a subset $A$ of $\mathbf{R}^{n}$. For $a \in A$,

$$
T_{a}^{m} F=\sum_{|k| \leq m} \frac{(x-a)^{k}}{k !} F^{k}(a)
$$

is the Taylor polynomial of $F$ in $a$ and $J^{m}\left(T_{a}^{m} F\right)$ is the $m$-jet on $A$ given by the derivatives of the smooth function $T_{a}^{m} F$. If we set $R_{a}^{m} F=F-J^{m}\left(T_{a}^{m} F\right)$, then $F$ will be a $C^{m}$ Whitney field if, and only if, for each $z \in R^{n}$,

$$
\left(R_{x}^{m} F(y)\right)^{k}=o\left(|x-y|^{m-|k|}\right) \quad \text { as } x, y \rightarrow z
$$

for $x, y \in A$ and $|k| \leq m$.

We also set, for $K \subseteq A$ compact,

$$
|F|_{m}^{K}=\max _{|k|=m}\left\{\sup _{K}\left|F^{k}(x)\right|\right\}
$$

(note that, contrary to usual practice, this seminorm only involves the $m$-th order derivatives).

If $A, B \subset \mathbf{R}^{n}$, then $d(A, B)=\inf _{x \in A, y \in B}|x-y|$. Also we let $D(A, B)=$ $\sup _{y \in B} d(A, y)$, which is finite if $B$ is compact.

We will use the Landau notation: if $f$ and $g$ are real-valued functions on $A \subset \mathbf{R}^{n}$, $a \in A$, then $g=O(f)$ at $a$ (respectively $g=o(f)$ at $a$ ) means that $|g / f|$ is bounded (respectively $|g / f| \rightarrow 0$ ) as $f(x) \rightarrow 0$ and $x$ is near $a$; furthermore, we say $g=O(f)$ (respectively $g=o(f)$ ) if it is so at each point of the domain $A$ of $f$ and $g$.

In all of the following, $S \subset V$ will be closed subsets of $\mathbf{R}^{n}, 0 \in S$, such that

1. $M=V \backslash S$ is a $C^{m}$ submanifold, with $m \geq 1$;

2. $V$ and $S$ are path connected;

3. $V=\bar{M}$. 
For $x, y \in V, \delta(x, y)$ denotes the geodesic distance on $V$ between $x$ and $y$, that is, the infimum of the lengths of all summable curves in $V$ joining $x$ to $y$ (possibly $\delta(x, y)=\infty$; for instance if one forms $V$ by rotating the curve $y=2 x+x \sin (1 / x)$, $x \geq 0$, about the $x$-axis, then $\delta(x, y)$ is finite for all points $x, y \in M$, but $\delta(x, 0)=\infty$ for all $x \in M)$. Note that a curve joining $x$ to $y$ of length $\delta(x, y)$ may have to pass through $S$ : this is the case for the points $(0,1,0)$ and $(0,-1,0)$ in $V=\{(x, y, z) \in$ $\left.\mathbf{R}^{3}: x^{2}-y^{2} z-z^{3}=0\right\}$. Let $\delta(x, S)$ denote $\inf _{y \in S} \delta(x, y)$ and let $\delta^{\prime}(x, y)$ denote the smaller of $\delta(x, y)$ and $\delta(x, S)+\delta(y, S)$. First we look at the relations between some conditions we will use in the Generalized Hestenes' Lemmas.

Lemma I-1. Given $l>0$, the following are equivalent:

(a) $\delta^{\prime}(x, y)=O\left(\max \{d(x, S), d(y, S)\}^{l}\right), x, y \in M$,

(b) $\delta(x, S)=O\left(d(x, S)^{l}\right), x \in M$.

Furthermore, (a) and (b) are implied by:

(c) $\delta(x, y)=O\left(|x-y|^{l}\right), x, y \in M$.

Finally, if any of these conditions hold, then $l \leq 1$ and $\delta^{\prime}(x, y)<\infty$ for all $x, y \in V$.

Proof. $(b) \Longrightarrow(a)$ : There exists $C>0$ such that, for $x$ and $y$ sufficiently near 0 ,

$$
\delta^{\prime}(x, y) \leq \delta(x, S)+\delta(y, S) \leq C\left(d(x, S)^{l}+d(y, S)^{l}\right) \leq 2 C \max \{d(x, S), d(y, S)\}^{l} .
$$

$(a) \Longrightarrow(b)$ : We are given $x \in M$. Pick $y_{i} \in M$ such that $\left|y_{i}\right| \leq d(x, S) / 2^{i / l}$. There exist curves $\gamma_{i}$ in $V$ such that $\gamma_{0}$ starts at $x$, ends at $y_{1}$ and has length (outside $S$ ) less than $2 \delta^{\prime}\left(x, y_{1}\right)$ and, for $i>0, \gamma_{i}$ starts at $y_{i}$, ends at $y_{i+1}$ and has length (outside $S$ ) less than $2 \delta^{\prime}\left(y_{i}, y_{i+1}\right)$. Then the part outside $S$ of the path obtained by connecting the $\gamma_{i}$ 's has finite length $l$. There is a $C>0$ such that

$$
\begin{aligned}
\delta(x, S) \leq \ell & \leq 2 \delta^{\prime}\left(x, y_{1}\right)+\sum 2 \delta^{\prime}\left(y_{i}, y_{i+1}\right) \\
& \leq 2 C\left(\delta(x, S)^{l}+\sum d\left(y_{i}, S\right)^{l}\right) \\
& \leq 2 C\left(\delta(x, S)^{l}+\sum \frac{\delta(x, S)^{l}}{2^{i}}\right) \leq 4 C \delta(x, S)^{l} .
\end{aligned}
$$

$(c) \Longrightarrow(a)$ : Indeed,

$$
\delta^{\prime}(x, y) \leq \delta(x, S)+\delta(y, S) \leq d(x, S)^{l}+d(y, S)^{l} \leq C(2 \max \{d(x, S), d(y, S)\})^{l} .
$$

To prove the last statement of the lemma, suppose $l>1$ in $(b)$. Then, for $d(x, S)$ sufficiently small, $d(x, S) \leq \delta(x, S) \leq C d(x, S)^{l}<d(x, S)$, yielding a contradiction.

Definition I-1. An $m$-Taylor field $F$ on $V$ is said to be flat of order $c \geq 0$ at $S$ if:

1) $\left.F\right|_{M}$ is a $C^{m}$ Whitney field on $M$ and $\left.F\right|_{S}=0$;

2) $\left|F^{k}(x)\right|=o\left(d(x, S)^{c}\right)$ for any $k$ such that $|k|=m$.

Definition I-2. Let us define the following conditions for $x, y \in M$ near to $S$ :

(A) There exist $K>0,0<l \leq 1$ and $r \geq 1$ such that:

A1) $\delta(x, S)=O\left(d(x, S)^{l}\right)$;

$A 2)$ if $|x-y| \leq K \max \{d(x, S), d(y, S)\}^{r}$, then

$$
\delta(x, y)=O\left(|x-y| \max \{d(x, S), d(y, S)\}^{l-r}\right) .
$$

(B) There exist $K>0,0<l \leq 1$ and $r \geq 1$ such that:

B1) $\delta(x, y)=O\left(|x-y|^{l}\right)$; 
$B 2)$ if $|x-y| \leq K \max \{d(x, S), d(y, S)\}^{r}$, then

$$
\delta(x, y)=O\left(|x-y| \max \{d(x, S), d(y, S)\}^{r(l-1)}\right) .
$$

If $V$ fulfils $(A)$ (respectively $(B)$ ) we say that $V$ is $(r, l)$-regular of type A (respectively of type B). We shall generically say " $(r, l)$-regular" when the type does not need to be specified.

Evidently, $B 1 \Rightarrow A 1$ in Definition I-2. If $V$ is $(r, l)$-regular, we shall unless otherwise stated assume that $l$ is the largest number satisfying $A 1$ (respectively $B 1$ ) and $r$ is the smallest number satisfying $A 2$ (respectively $B 2$ ) using the above $l$. If $V$ is $(r, l)$-regular of type $\mathrm{B}$, we shall denote by $l^{\prime}$ the exponent for condition $A 1$ on $V$ : we have of course $l^{\prime} \geq l$.

We set

$$
c_{m}(r, l)=\frac{(r-l) m}{l} .
$$

Moreover, if $V$ is $(r, l)$-regular of type $\mathrm{B}$ we let

$$
k_{m}(r, l)=\frac{(r-r l) m}{l^{\prime}} .
$$

The conditions described in Definition I-2 will allow us to prove a generalization of Hestenes' Lemma. Since they may appear quite technical, we will make a few comments about them before going on.

Clearly, $A 1$ and $B 1$ are global comparisons between euclidean and geodesic distance on $M$. We point out that, in Tougeron's terminology, $B 1$ means that $V$ is " $\frac{1}{l}$-regular". Other authors, e.g. [St], consider a more general version of this property called "the Whitney condition".

On the other hand, the two distances are always locally comparable in the above sense, and we can interpret $A 2$ and $B 2$ as giving an estimate of the diameters of the neighbourhoods where comparability holds. For instance, we give the following geometric interpretation of condition $A 2$ (analogous arguments hold for $B 2$ ).

For each $C>1$ and $x \in M$, there is a maximal positive number $\epsilon(x, C)$ such that $|x-y| \leq \epsilon(x, C) \Rightarrow \delta(x, y) \leq C|x-y| \max \{d(x, S), d(y, S)\}^{l-r}$ for $y \in M$. This gives, for $x, y \in M$,

$$
|x-y| \leq \max \{\epsilon(x, C), \epsilon(y, C)\} \Rightarrow \delta(x, y) \leq C|x-y| \max \{d(x, S), d(y, S)\}^{l-r} .
$$

Hence condition $A 2$ is equivalent to saying that there are $C, K, r$ such that $\epsilon(x, C) \geq K d(x, S)^{r}$ for $x$ near $S$.

Because there is considerable interest in condition $B 1$, we include the following result, although it is not used anywhere else in the paper.

Lemma I-2. If $S=\{0\}$, then $A 1$ and $A 2$ imply $B 1$ with exponent $l / r$.

Proof. On $\left\{|x-y| \leq K \max \{|x|,|y|\}^{r}\right\}, \delta(x, y) \leq C|x-y| \max \{|x|,|y|\}^{l-r}$, so

$$
\frac{\delta(x, y)}{|x-y|^{l / r}} \leq C|x-y|^{(r-l) / r} \max \{|x|,|y|\}^{l-r} \leq C K^{(r-l) / r} .
$$

On $\left\{|x-y| \geq K \max \{|x|,|y|\}^{r}\right\}, A 1$ implies

$$
\delta(x, y)=O\left(\max \{|x|,|y|\}^{l}\right)=O\left(|x-y|^{l / r}\right) .
$$


We finally remark that, if $V$ is 1-regular, we can take $r=l=1$ and so $c_{m}=$ $k_{m}=0$.

Generalized Hestenes' Lemma. Suppose that $V$ is $(r, l)$-regular and that $F$ is an $m$-Taylor field on $V$ which is flat of order $c$ at $S$. Assume that $c \geq c_{m}(r, l)$ if $V$ is of type $A$ or that $c \geq k_{m}(r, l)$ if $V$ is of type B. Then $F$ is a $C^{m}$ Whitney field on $V$.

Proof. By the definition of a Whitney field and our assumptions on $F$, it suffices to prove that, for each $k$ with $|k| \leq m$,

$$
\frac{\left|\left(R_{x}^{m} F\right)^{k}(y)\right|}{|x-y|^{m-|k|}} \rightarrow 0
$$

as $x$ and $y$ tend to a point of $S$.

Let $\Delta$ be a curve in $V$ from $x$ to $y$ such that $\Delta=\Delta_{1} \cup \Delta_{2}, \Delta_{1}$ of length less than $2 \delta^{\prime}(x, y), \Delta_{2} \subset S$, and

$$
D(S, \Delta) \leq \max \{d(x, S), d(y, S)\}+2 \delta^{\prime}(x, y)=O(\max \{\delta(x, S), \delta(y, S)\}) .
$$

Since $\Delta$ is compact, the argument of IV.2.5.1 of [T] (p. 76) implies that

$$
\frac{\left|\left(R_{x}^{m} F\right)^{k}(y)\right|}{|x-y|^{m-|k|}}=O\left(\left(\frac{\delta^{\prime}(x, y)}{|x-y|}\right)^{m-|k|} \cdot|F|_{m}^{\Delta}\right)
$$

(whereas in $[\mathrm{T}]$, one uses $\delta(x, y)$, we can get the same result using $\delta^{\prime}(x, y)$ since the Taylor field is identically 0 on the part of our curves contained in $S$ ). By our assumption on $F$ and (1),

$$
|F|_{m}^{\Delta}=o\left(D(S, \Delta)^{c}\right)=o\left(\max \{\delta(x, S), \delta(y, S)\}^{c}\right) .
$$

Thus,

$$
\frac{\left|\left(R_{x}^{m} F\right)^{k}(y)\right|}{|x-y|^{m-|k|}}=o\left(\left(\frac{\delta^{\prime}(x, y)}{|x-y|}\right)^{m-|k|} \cdot \max \{\delta(x, S), \delta(y, S)\}^{c}\right) .
$$

By Lemma I-1, we have

$$
\frac{\left|\left(R_{x}^{m} F\right)^{k}(y)\right|}{|x-y|^{m-|k|}}=o\left(\left(\frac{\delta^{\prime}(x, y)}{|x-y|}\right)^{m-|k|} \cdot \max \{d(x, S), d(y, S)\}^{l c}\right) .
$$

We deal now with types A and B separately.

\section{Type A.}

Case I. $|x-y| \leq K \max \{d(x, S), d(y, S)\}^{r}$.

Since $\delta^{\prime} \leq \delta$, from $A 2$ and (3) we have

$$
\begin{aligned}
\frac{\left|\left(R_{x}^{m} F\right)^{k}(y)\right|}{|x-y|^{m-|k|}} & =o\left(\left(\max \{d(x, S), d(y, S)\}^{l-r}\right)^{m-|k|} \cdot \max \{d(x, S), d(y, S)\}^{l c}\right) \\
& =o\left(\max \{d(x, S), d(y, S)\}^{(l-r)(m-|k|)+l c}\right) \\
& =o\left(\max \{d(x, S), d(y, S)\}^{(r-l)|k|}\right)
\end{aligned}
$$

since $c \geq c_{m}(r, l)$.

Case II. $|x-y| \geq K \max \{d(x, S), d(y, S)\}^{r}$. 
By Lemma I-1, $A 1$ and (3),

$$
\begin{aligned}
\frac{\left|\left(R_{x}^{m} F\right)^{k}(y)\right|}{|x-y|^{m-|k|}} & =o\left(\left(\frac{\max \{d(x, S), d(y, S)\}^{l}}{\max \{d(x, S), d(y, S)\}^{r}}\right)^{m-|k|} \cdot \max \{d(x, S), d(y, S)\}^{l c}\right) \\
& =o\left(\max \{d(x, S), d(y, S)\}^{(l-r)(m-|k|)+l c}\right),
\end{aligned}
$$

and we conclude as above.

\section{Type B.}

Case I. $|x-y| \leq K \max \{d(x, S), d(y, S)\}^{r}$.

By $B 2, \delta^{\prime} \leq \delta$ and (3),

$$
\begin{aligned}
\frac{\left|\left(R_{x}^{m} F\right)^{k}(y)\right|}{|x-y|^{m-|k|}} & =o\left(\left(\max \{d(x, S), d(y, S)\}^{r l-r}\right)^{m-|k|} \cdot \max \{d(x, S), d(y, S)\}^{l c}\right) \\
& =o\left(\max \{d(x, S), d(y, S)\}^{(r l-r)(m-|k|)+l c}\right) \\
& =o\left(\max \{d(x, S), d(y, S)\}^{(r-r l)|k|}\right)
\end{aligned}
$$

since $c \geq k_{m}(r, l)$.

Case II. $|x-y| \geq K \max \{d(x, S), d(y, S)\}^{r}$.

By $B 1$

$$
\begin{aligned}
\frac{\left|\left(R_{x}^{m} F\right)^{k}(y)\right|}{|x-y|^{m-|k|}} & =o\left(|x-y|^{(l-1)(m-|k|)} \cdot \max \{d(x, S), d(y, S)\}^{l c}\right) \\
& =o\left(\max \{d(x, S), d(y, S)\}^{r(l-1)(m-|k|)+l c}\right) \\
& =o\left(\max \{d(x, S), d(y, S)\}^{r(1-l)|k|}\right) .
\end{aligned}
$$

In what follows, "GHL" will stand for "Generalized Hestenes' Lemma".

Remarks. (1) Assume $V$ 1-regular: by the last remark before GHL the assumption on $F$ in GHL becomes that $F(x) \rightarrow 0$ as $x \rightarrow 0$. Thus we recover the usual Hestenes' Lemma, which is the case $V=\mathbf{R}^{n}$.

(2) Also see the generalized Hestenes' Lemma of [BiMP]. They have a very nice and simple result using only the exponent of $l$-regularity. However, their hypothesis is not on the order of flatness of the $m$-th order terms; rather that the Whitney field be of prescribed higher order of differentiability with continuous derivatives where it is not known to be Whitney.

Examples. Let $r_{A}, l_{A}, r_{B}$ and $l_{B}$ denote the numbers of Definition I-2, parts A and $\mathrm{B}$, respectively.

I-1. The "elliptical bugle".

Let $M$ be

$$
\frac{y^{2}}{x^{2 a}}+\frac{z^{2}}{x^{2 b}}=1, \quad 0<a \leq b, \quad x>0
$$

and let $V=M \cup\{0\}$. Assume $1 \leq a<b$. Then $l_{A}=1, l_{B}=a / b, r_{A}=b-a+1$ and $r_{B}=b$.

That $l_{A}=1$ follows from Proposition I-4, since $V$ is a semialgebraic set with isolated singularity. 
For each $x>0$ and for each $-x^{a}<y<x^{a}$, let $p=p(x, y)$ and $q=q(x, y)$ be the points on $M$ which project to $(x, y, 0)$. Then $|p-q|=2 z=2 x^{b-a} \sqrt{x^{2 a}-y^{2}}$ and, for $x$ small, $x^{b} \ll x^{a}$, so $\delta(p, q)=O(\max \{z, u\})$, where $u=x^{a}-y$.

$\delta(p, q) /|p-q|$ is greatest when $y=0$, so we restrict to $p=p(x, 0)$ and $q=q(x, 0)$. Then $\delta(p, q)=O\left(x^{a}\right)$ and $|p-q|=2 x^{b}$. Thus $\delta(p, q)=O\left(|p-q|^{a / b}\right)$; hence $l_{B}=a / b$.

We want $r=r_{B}$ to satisfy

$$
|p-q|=O\left(x^{r}\right) \Longrightarrow \delta(p, q)=O\left(|p-q| x^{r_{B}(l-1)}\right) .
$$

(We omit the proof that this forces the same inequality to hold for $p$ and $q$ replaced by arbitrary $P$ and $Q$ in $M$, and $x$ replaced by $\max \{P, Q\}$.) So we want $z=O\left(x^{r}\right)$ to imply $\max \left\{\frac{u}{z}, 1\right\}=O\left(x^{r\left(l_{B}-1\right)}\right)$, i.e. $\frac{u}{z}=O\left(x^{r\left(l_{B}-1\right)}\right)$, since $r\left(l_{B}-1\right)<0$. Replacing $y$ by $x^{a}-u$ in $y=x^{a-b} \sqrt{x^{2 b}-z^{2}}$, we get

$$
\frac{1}{2} z^{2} x^{a-2 b} \leq u=x^{a}-x^{a} \sqrt{1-\left(\frac{z}{x^{b}}\right)^{2}} \leq z^{2} x^{a-2 b} .
$$

Thus we want $z=O\left(x^{r}\right)$ to imply that $z x^{a-2 b}=O\left(x^{r\left(l_{B}-1\right)}\right)$, which holds if $z=O\left(x^{\frac{r}{b}(a-b)+2 b-a}\right)$. Thus we need to have $r \geq \frac{r}{b}(a-b)+2 b-a$, that is, $r \geq b$. Thus we can take $r_{B}=b$.

Now let $r$ denote $r_{A}$. So we need that

$$
|p-q| \leq x^{r} \Longrightarrow \delta(p, q)=O\left(|p-q| x^{1-r}\right) .
$$

Calculating as above, we get that the right hand side holds if $z=O\left(x^{1-r+2 b-a}\right)$. Now, on all of $V, z=O\left(x^{b}\right)$, and $b=1-r+2 b-a$ for $r=b-a+1$, so we are done.

Substituting the values of $r_{A}, r_{B}, l_{A}, l_{B}$ in the formulas defining $c_{m}$ and $k_{m}$, we get $c_{m}=k_{m}=(b-a) m$.

I-2. Let $x_{n}=1 / n$ and let $f(x)=x^{3 / 2}$. In the $x y$-plane, join the points $\left(x_{2 n}, 0\right)$ to the points $\left(x_{2 n+1}, f\left(x_{2 n+1}\right)\right)$ by straight line segments, and similarly join the latter points to $\left(x_{2 n+2}, 0\right)$. Round off at the sharp points and include 0 to form a curve $V$. An elementary calculation shows the $l_{A}=l_{B}=1 / 2$, and $r_{A}=r_{B}=1$. So $c_{m}=k_{m}=m$.

We can get a surface with the same $r$ 's and $l$ 's by revolving this curve about any line through 0 different from the $x$-axis.

We know of no example in which $B$ gives different estimates on the order of flatness than $A$; however the $l$-regularity assumption used there is a frequently studied quantity, so we feel justified in including this method.

Next we give an example proving that conditions $A 2$ and $B 2$ are needed to get our result.

Example I-3. "Flat elliptical bugle".

Let $\phi(x)$ be a smooth function, positive when $x>0$, and infinitely flat at 0 . Let $M$ be

$$
\frac{y^{2}}{\phi^{2}}+\frac{z^{2}}{\phi^{4}}=1, \quad x>0
$$

and let $V=M \cup\{0\}$.

For each $x>0$, let $p=p(x)$ and $q=q(x)$ be the points $\left(x, 0, \pm \phi^{4}\right)$ on $M$. Then $|p-q|=2 \phi(x)^{2}$ and $\delta(p, q) \sim 2 \phi(x)$ (i.e. $\delta(p, q)=O(\phi(x))$ and $\left.\phi(x)=O(\delta(p, q))\right)$. Since the discrepancy between $\delta$ and euclidean distance is greatest for these points 
$p, q$, we conclude that condition $B 1$ holds with $l=\frac{1}{2}$. On the other hand $|p-q|=$ $O\left(x^{r}\right)$ holds on all of $M$ (sufficiently near 0 ) for any $r$, and

$$
\delta(p, q) /|p-q| \sim \frac{1}{\phi}
$$

so $B 2$ fails for every $r$.

Let $\alpha(y)$ be a smooth function satisfying $0 \leq \alpha \leq 1, \alpha=1$ on $|y| \leq \frac{1}{3}, \alpha=0$ on $|y| \geq \frac{2}{3}$, and $\left|\alpha^{\prime}\right| \leq C$. Pick an $s, 1<s<2$, and define for $x>0$

$$
f(x, y, z)=\phi(x)^{s} \alpha(y / \phi(x)) .
$$

By an easy calculation, $\partial f / \partial y$ is $O\left(\phi^{s-1}\right)$, as is $\partial f / \partial x$ on $|y| \leq \phi(x)$, hence on $M$. Thus the 1-jet of $f$ on $M$ is infinitely flat at 0 . Let $F$ be the 1 -jet field of $f$ restricted to $M^{+}=M \cap\{z \geq 0\}$ and of $-f$ restricted to $M^{-}=M \cap\{z \leq 0\}$. (F is identically 0 on a neighborhood of $M^{+} \cap M^{-}$.) $F$ is a $C^{1}$ Whitney field on $M$ with coefficients infinitely flat at 0 , but it has no $C^{1}$ extension near 0 since

$$
\frac{f(p)-f(q)}{|p-q|}=\phi^{s-2} \rightarrow \infty
$$

We conclude that condition $B 1$ is not strong enough by itself to give the type of extendibility result we are after.

Next, using a result which has not yet been published, we prove that our results hold when we assume $V$ to be subanalytic and $S$ to be compact.

Proposition I-1. If $V$ is subanalytic and $S$ is bounded, then $V$ is $(r, l)$-regular.

Proof. $S$ is compact by our assumption. Condition $B 1$ of Definition I-2 holds locally for any subanalytic set ("Whitney condition", [St]), hence $B 1$ is fulfilled in our case. We show that conditions $A 2$ and $B 2$ hold on $M$ by proving a stronger result. By a recent result of Kurdyka and Orro ([KO]), there is a subanalytic metric function on $V$ which is equivalent to $\delta$. Since the equivalence will preserve the constructions we do below, without loss of generality we assume that $\delta$ is subanalytic. Let $C>1$ and let $\Delta$ be the diagonal in $M \times M=M^{2}$. Then the set $U=\left\{(x, y) \in M^{2}: \delta(x, y)\right.$ $<C|x-y|\} \cup \Delta$ is open and subanalytic in $V^{2}$. Moreover, each $x \in M$ has a neighborhood $N$ in $M$ such that $y, z \in N$ implies $\delta(z, y)<C|z-y|$. Then $N \times N \subseteq U$ is a neighborhood of $(x, x)$.

Let $U^{\prime}=V^{2} \backslash U$ and let $\alpha: \mathbf{R}^{n} \times \mathbf{R}^{n} \rightarrow \mathbf{R}$ be defined as the distance of $(x, y)$ from $U^{\prime}$. Then $\alpha$ is subanalytic and positive on $\Delta$. By the Lojasiewicz inequality, there are positive constants $K$ and $r$ such that $\alpha(x, x) \geq K d(x, S)^{r}$ (since $\left.S \times V \cup V \times S \subset U^{\prime}\right)$.

Since $d((x, y),(x, x))=|y-x|$, thus $|x-y|<K d(x, S)^{r}$ implies $(x, y) \in U$, that is, $\delta(x, y)<C|x-y|$.

We shall give some further results in the simplified case of an isolated singularity. Therefore we shall assume for the rest of this section that $S=\{0\}$. Under this hypothesis, we introduce another regularity condition which is a particular case of Bekka and Trotman's $(a+\delta)$-regularity $([\mathrm{BT}])$ and which is weaker than Whitney's $b$-regularity. If $x \in M$, we shall denote by $P_{x}: \mathbf{R}^{n} \rightarrow T_{x} M$ the orthogonal projection, where $T_{x} M$ is considered a subspace of $\mathbf{R}^{n}$ through 0 .

Definition I-3. $V$ is said to be $\delta$-regular if there are $r>0, \delta \in(0,1)$ such that, for any $x \in M \cap B(0, r)$, the inequality $\delta|x| \leq\left|P_{x} x\right|$ holds. 
Let $\theta$ denote the angle between $T_{x} M$ and $\overrightarrow{0 x}$. Then $\cos \theta=\left|P_{x} x\right| /|x|$, so the above condition gives a positive lower bound on $\cos \theta$, hence an upper bound on $\theta$ smaller than $\pi / 2$.

The proof of a more general version of the next proposition can be found in [B].

Proposition I-2. If $V$ is $\delta$-regular, the germ of $M$ at any point of $\mathbf{R}^{n}$ has a finite number of connected components.

Proposition I-3. Let us assume that $V$ is $\delta$-regular; then $l=1$ (where $l$ is the coefficient of condition A1).

Proof. Let us consider the smooth vector field on $M$ given by

$$
\xi_{x}=-P_{x}\left(\frac{x}{|x|}\right) /\left|P_{x}\left(\frac{x}{|x|}\right)\right|^{2} .
$$

Then $\xi \neq 0$ on $B(0, r)$. Fix $x \in B(0, r)$ and denote by $\alpha(t)$ the integral curve of $\xi$ with $\alpha(0)=x$. Then $\alpha \in C^{0}((-\epsilon,|x|], V) \cap C^{1}((-\epsilon,|x|), M)$ for a suitable $\epsilon>0$, and $|\alpha(t)|=-t+|x|$. The length of $\alpha$ is $\int_{0}^{|x|}\left|\alpha^{\prime}(t)\right| d t \leq \frac{1}{\delta}|x|$. Thus $l=1$.

We recall that, if $V$ is subanalytic with an isolated singularity, then it is $\delta$ regular (in fact, by [Hi], the stratification $\{M,\{0\}\}$ of $V$ is $b$-regular in the sense of Whitney), so $l=1$.

We now denote by $\delta_{M}$ the geodesic distance on $M . \delta_{M}$ is always finite and we have $\left.\delta\right|_{M} \leq \delta_{M}$, but it is possible to find examples in which $\left.\delta\right|_{M} \neq \delta_{M}$. If $\delta=\delta_{M}$, then we can also consider in GHL $m$-Taylor fields on $M$ which are not a priori continuous at $S$, since this hypothesis was only used to apply the mean value theorem. If our paths remain in $M$, this is not a problem. Actually this hypothesis is very natural if $M$ is connected, and it is satisfied in the subanalytic case, as the next proposition shows.

Proposition I-4. Let $V$ be subanalytic. Assume that the germ of $M$ at 0 is connected. Then $\delta=\delta_{M}$.

Proof. Since $\delta \leq \delta_{M}$, it is enough to prove the following:

Assertion. Let $I=[0,1]$. If $x, y \in M$, let $\gamma: I \rightarrow V$ be a continuous arc such that:

1) $\gamma(0)=x, \gamma(1)=y, \gamma\left(t_{0}\right)=0$ for a $t_{0} \in I$ and $\gamma(t) \in M$ for any $t \in I \backslash\left\{t_{0}\right\}$;

2) $\gamma$ is piecewise differentiable in $M$ and has finite length $\ell(\gamma)$.

Then there is a sequence $\left\{\gamma_{k}\right\}$ of piecewise differentiable arcs in $M$ such that

$$
\lim _{k \rightarrow \infty} \ell\left(\gamma_{k}\right)=\ell(\gamma)
$$

To prove this assertion we shall apply a result of Stasica ([St]). From Stasica's work we may state that there exists an open neighborhood $U$ of 0 in $V$ such that:

i) $U \backslash\{0\}=\bigcup_{j=1}^{p} U_{j}$;

ii) any $U_{j}$ is open in $V$ and $0 \in \bar{U}_{j}$;

iii) there are $C>0$ and $l>0$ (which can chosen to be independent of $j$ ) such that, if $x, y \in U_{j}$, then there is a smooth $\operatorname{arc} \alpha: I \rightarrow U_{i}$ with $\ell(\alpha) \leq C|x-y|^{l}$.

Now let $\left\{x_{k}\right\}$ (respectively $\left\{y_{k}\right\}$ ) be a sequence of points in $\gamma\left(\left(0, t_{0}\right)\right)$ (respectively $\left.\gamma\left(\left(t_{0}, 1\right)\right)\right)$ converging to 0 . The assertion will be proved if we show that there is a sequence $\left\{\alpha_{k}\right\}$ of piecewise differentiable $\operatorname{arcs}$ in $M$ such that $\alpha_{k}$ joins $x_{k}$ to $y_{k}$ and $\ell\left(\alpha_{k}\right)$ goes to 0 as $k$ goes to $\infty$. 
Let $B^{\epsilon}=\{x:|x|<\epsilon\}, M^{\epsilon}=B^{\epsilon} \cap M$ and $U_{j}^{\epsilon}=B^{\epsilon} \cap U_{j}$. Since the germ of $M$ at 0 is connected, there is an $\epsilon_{0}>0$ such that, for all $\epsilon \leq \epsilon_{0}, M^{\epsilon}$ is connected. Pick a $k_{0}$ such that $\epsilon_{k}=\max \left\{\left|x_{k}\right|,\left|y_{k}\right|\right\}<\epsilon_{0}$ for all $k \geq k_{0}$. Fix such a $k$. If there is a $j$ such that $x_{k}$ and $y_{k}$ are in the same $U_{j}$, then we take $\alpha_{k}$ as in iii) above. Otherwise there are $j$ and $j^{\prime}$ with $j \neq j^{\prime}$ such that $x_{k} \in U_{j}$ and $y_{k} \in U_{j^{\prime}}$. Since $M^{\epsilon_{k}}$ is connected and is covered by the $U_{1}^{\epsilon_{k}}, \ldots, U_{p}^{\epsilon_{k}}$, we can find $q \leq p+1$ points $z_{1}, \ldots, z_{q}$ such that:

1) $z_{1}=x_{k}, z_{q}=y_{k}$

2) there exist $h_{1}, \ldots, h_{q}$ such that for $i=1, \ldots, q-1, z_{i}$ and $z_{i+1}$ are in the same $U_{h_{i}}^{\epsilon_{k}}$.

For each $i$ we have an $\operatorname{arc} \alpha_{k}^{i}$ in $U_{h_{i}}^{\epsilon_{k}}$ joining $z_{i}$ to $z_{i+1}$ of length $\ell\left(\alpha_{k}^{i}\right) \leq$ $C\left|z_{i}-z_{i+1}\right|^{l} \leq C\left(2 \epsilon_{k}\right)^{l}$. Hence the arc $\alpha_{k}$ obtained by gluing the $\alpha_{k}^{i}$ 's has length less than $C^{\prime} \epsilon_{k}^{l}$.

\section{An EXTENSION RESUlt}

Let $V$ be as in section I with $M$ of class $C^{m+1}$ at least, and let $f: V \rightarrow \mathbf{R}$ be a continuous function such that $\left.f\right|_{M} \in C^{m}(M, \mathbf{R})$ and $\left.f\right|_{S} \equiv 0$.

We want to study the possibility of extending $f$ to a $C^{m}$ function on $\mathbf{R}^{n}$ by means of the GHL. In the trivial case $V=\mathbf{R}^{n}$, if all derivatives of $f$ tend to 0 as $\delta(x, S)$ does, then $f$ is $C^{m}$ on $\mathbf{R}^{n}$ ( $m$-flat) by Hestenes' Lemma. So, we have to deal with the notion of "flatness" on $V$. If $V$ were smooth at $S$, the meaning of " $f$ is $m$-flat at $S$ " would be clear: just take local coordinates of $V$ at any point of $S$ and compute the derivatives of $f$ up to order $m$, requiring that they vanish on $S$. This notion is independent of the choice of local coordinates, though the derivatives are not. In the singular case, one should have a limit condition, such as "all derivatives tend to 0", but this notion is no longer intrinsic, as is illustrated by the following:

Example. Let $V=\left\{(x, y): y^{2}=x^{3}, x \geq 0\right\} \subset \mathbf{R}^{2}$ and $f(x, y)=y$. If we parametrize $V$ as $x=t^{2}, y=t^{3}$, then $f$ is 2-flat with respect to this parametrization, while it is just 1-flat if we use the parametrization $x=u, y=u^{\frac{3}{2}}$.

In [Wi], an extension result for functions which are "flat" with respect to a fixed parametrization is obtained as a byproduct of a different theory.

Here we try to define an intrinsic notion of flatness. Let us consider the 1-form $d f$ on $M$; since $d f$ is a tensor on $M$, we can consider its iterated generalized covariant derivatives with respect to the Levi-Civita connection $\nabla$ on $M$ induced by the standard connection $D$ on $\mathbf{R}^{n}$. Then we may set:

$$
\nabla^{r} f=\overbrace{\nabla \ldots \nabla}^{r-1 \text { times }} d f
$$

for $r>1, \nabla^{1} f=d f$ and $\nabla^{0} f=f$.

We say that $f$ is $m$-flat at 0 if, for $r \leq m$, we have $\lim _{x \rightarrow 0}\left\|\nabla^{r} f\right\|(x)=0$, where $\|\cdot\|$ is the norm for covariant tensors.

The extension theorems we will get in this section and in the next one need a stronger notion of flatness for $f$. On the other hand, we shall actually obtain extensions of an $m$-jet on $M$ which depends on $f$. Since the component with index 0 of this jet is $f$ itself, the result is stronger than a plain extension of $f$ and is tied to the way of extending $f$ on a neighborhood of $M$ in $\mathbf{R}^{n} \backslash S$. 
We consider the standard orthogonal tubular neighborhood of $M$ in $\mathbf{R}^{n} \backslash S$. This is an open neighborhood $T$ of $M$ in $\mathbf{R}^{n} \backslash S$ on which the map $\pi: T \rightarrow M$ which sends each point to the nearest point on $M$ is defined and smooth. An idea is to take the function $F=f \circ \pi \in C^{m}(T, \mathbf{R})$, to compute the $m$-jet of $F$ on $M$ and to look for conditions on $f$ so that this jet fulfils the hypotheses of the GHL. In this way, the differential geometry of $M$ as a submanifold is involved. In this section we carry on this work in the case $m=2$, which turns out to be the case in which we get the most meaningful conditions.

Remark. Wall, in [W], gives arguments which essentially deal with these same issues.

From now on $e_{1}, \ldots, e_{n}$ will be the canonical basis in $\mathbf{R}^{n}$ and $\operatorname{dim} M=d$. Recalling that $D_{v} F=v(F)$ are the derivatives of $F$ in the direction $v$, we set $D_{i}=D_{e_{i}}$.

We recall some details about $T$. Let $p=n-d=\operatorname{codim} M$ and let $N$ be a normal unit vector field on $M$. Then the operator $L_{N} \in \operatorname{End}(T M)$ defined as $L_{N, x}(v)=P_{x} D_{v} N$ for $x \in M$ and $v \in T_{x} M$ is self-adjoint and smooth (we recall that $P_{x}: \mathbf{R}^{n} \rightarrow T_{x} M$ is the orthogonal projection). The eigenvalues of $L_{N, x}$ are the negatives of the principal curvatures of $M$ at $x$ in direction $N(x)$; we denote these principal curvatures by $\kappa_{1}^{N}(x), \ldots, \kappa_{d}^{N}(x)$. Then we can define $\kappa^{N}(x)=\max \left\{\left|\kappa_{h}^{N}(x)\right|: 1 \leq h \leq d\right\}$ and $\kappa(x)=\underset{N}{\sup \kappa^{N}}(x)$. It is immediate that

$$
\left\|L_{N, x}\right\|=\kappa^{N}(x) \leq \stackrel{N}{\kappa}(x) .
$$

As usual (see e.g. [Mi]), we take

$$
T \subseteq\left\{x \in \mathbf{R}^{n} \backslash\{0\}:|x-\pi(x)|<\kappa(\pi(x))^{-1}\right\} .
$$

Now, if $x \in T$, we have $D_{i} F(x)=d_{\pi(x)} f \cdot d_{x} \pi e_{i}$. Then, by construction, $d_{x} \pi=P_{x}$ for $x \in M$ and

$$
D_{i} F(x)=d_{x} f \cdot P_{x}\left(e_{i}\right) .
$$

We compute $D_{i} D_{j} F$ on $M$.

Lemma II-1. Let $T$ be the orthogonal tubular neighborhood of $M$ in $\mathbf{R}^{n} \backslash\{0\}$. Then, if $x \in T$,

$$
d_{x} \pi=\Delta_{x} \circ P_{\pi(x)},
$$

where $\Delta_{x}$ is the self-adjoint automorphism of $T_{\pi(x)} M$ which, for a local orthonormal frame $\nu=\left\{N_{1}, \ldots, N_{p}\right\}$ of normal smooth vector fields on $M$, is given by

$$
\Delta_{x}=\left(I+\sum_{i=1}^{p} \rho_{i}(x) L_{i, \pi(x)}\right)^{-1}
$$

where $\rho_{i}(x)=\left\langle x-\pi(x), N_{i}(\pi(x))\right\rangle$ and $L_{N_{i}}=L_{i}$.

Proof. Let us fix a frame $\nu=\left\{N_{1}, \ldots, N_{p}\right\}$. If $x \in T, x-\pi(x) \in\left(T_{\pi(x)} M\right)^{\perp}$. Hence

$$
x=\pi(x)+\sum_{i=1}^{p}\left\langle x-\pi(x), N_{i}(\pi(x))\right\rangle N_{i}(\pi(x)) .
$$

Let $v \in T_{x} \mathbf{R}^{n}$ and let us consider the smooth curve $\alpha(t)=\pi(t v+x)$ on $M$, which is defined in a neighborhood of 0 . Then $\alpha$ fits $d_{x} \pi v$, that is, $\alpha(0)=\pi(x)$ and 
$\alpha^{\prime}(0)=d_{x} \pi v$. Differentiating both sides of the last displayed equation in direction $v$ and evaluating at $x$, we get

$$
\begin{aligned}
v & =d_{x} \pi v+\sum_{i=1}^{p} \rho_{i}(x) D_{d_{x} \pi v} N_{i} \\
& +\left\langle v, N_{i}(\pi(x)\rangle N_{i}(\pi(x))+\left\langle x-\pi(x), D_{d_{x} \pi v} N_{i}\right\rangle N_{i}(\pi(x)) .\right.
\end{aligned}
$$

Then

$$
P_{\pi(x)}=\Lambda_{x} d_{x} \pi
$$

where

$$
\Lambda_{x}=I+\sum_{i=1}^{p} \rho_{i}(x) L_{i, \pi(x)} .
$$

Equation (5) yields that $\Lambda$ is independent of $\nu$ and surjective. Hence $\Lambda$ is nonsingular, and (4) follows.

For each $i \leq n$, we define a smooth vector field on $M$ with $P_{i}(x)=P_{x}\left(e_{i}\right)$.

Lemma II-2. If $i, j \leq n$ and $x \in M$, then, for any frame $\nu=\left\{N_{1}, \ldots, N_{p}\right\}$, we have

$$
\begin{array}{rl}
D_{i} D_{j} F(x)=\nabla^{2} & f\left(P_{i}(x) P_{j}(x)\right) \\
& -d f\left(\sum_{h=1}^{p}\left\langle N_{h}(x), e_{i}\right\rangle L_{h, x} P_{j}(x)+\left\langle N_{h}(x), e_{j}\right\rangle L_{h, x} P_{i}(x)\right) .
\end{array}
$$

Proof. For any $i \leq n$ and $x \in M$,

$$
e_{i}=P_{i}(x)+\sum_{h=1}^{p}\left\langle N_{h}(x), e_{i}\right\rangle N_{h}(x)
$$

Then

$$
D_{i} D_{j} F(x)=D_{P_{i}(x)} D_{j} F(x)+\sum_{h=1}^{p}\left\langle N_{h}(x), e_{i}\right\rangle D_{N_{h}(x)} D_{j} F(x) .
$$

Let $A$ and $B$ denote the first and the second summand respectively on the right side of $(7)$.

Computation of $A$. If $e_{i} \perp T_{x} M$, then $A=0$ trivially. Otherwise, let's consider the smooth curve $\alpha(t)=\pi\left(t e_{i}+x\right)$ on $x$, which is defined for $t$ near enough to 0 . Evidently $\alpha$ fits $P_{i}(x)$. Then, by the definition of generalized covariant derivative,

$$
\begin{aligned}
D_{P_{i}} D_{j} F(x) & =P_{i} P_{j} f(x) \\
& =\nabla d f\left(P_{i}(x), P_{j}(x)\right)+d f\left(\nabla_{P_{i}} P_{j}(x)\right) .
\end{aligned}
$$

Now

$$
P_{j}(\alpha(t))=e_{j}-\sum_{h=1}^{p}\left\langle N_{h}(\alpha(t)), e_{j}\right\rangle N_{h}(\alpha(t)) .
$$

Hence

$$
D_{P_{i}} P_{j}(x)=\sum_{h=1}^{p}-\left\langle N_{h}(x), e_{j}\right\rangle D_{P_{i}} N_{h}(x)-\left\langle e_{j}, D_{P_{j}} N_{h}(x)\right\rangle N_{h}(x)
$$


and, taking the tangential component,

$$
\nabla_{P_{i}} P_{j}(x)=\sum_{h=1}^{p}-\left\langle N_{h}(x), e_{j}\right\rangle L_{h, x} P_{i}(x) .
$$

Going back to (8), we get from (9)

$$
D_{P_{i}} D_{j} F(x)=\nabla d f\left(P_{i}(x), P_{j}(x)\right)-d f\left(\sum_{h=1}^{p}\left\langle N_{h}(x), e_{j}\right\rangle L_{h, x} P_{i}(x)\right) .
$$

Computation of $B$. We must compute $D_{N_{h}} D_{j} F$ for $h=1, \ldots, p, x \in M$. Let $h$ be fixed. The curve $\beta_{h}(t)=x+t N_{h}(x)$ fits $N_{h}(x)$, and $D_{j} F\left(\beta_{h}(t)\right)=d f \Delta_{\beta_{h}(t)} P_{j}(x)$ by Lemma II-1. Since $\pi\left(\beta_{h}(t)\right)=x$ and $\rho_{l}(\beta(t))=\delta_{l, h} t$ for $l=1, \ldots, p$, we have $\Delta_{\beta_{h}(t)}=\left(I+t L_{h, x}\right)^{-1}=\Delta(t)$. Now $\Delta^{-1}(t) \circ \Delta^{\prime}(t)+\left(\Delta^{-1}(t)\right)^{\prime} \circ \Delta(t) \equiv 0$ gives $\Delta^{\prime}(0)=-L_{h, x}$. Then

$$
D_{N_{h}} D_{j} F(x)=-d f\left(L_{h, x} P_{j}(x)\right) .
$$

Substituting (10) and (11) in (7), we obtain, for $x \in M$, formula (6).

Theorem II-1. Suppose that $V$ is $(r, l)$-regular and that $M$ is of class $C^{3}$ at least. Let $f: V \rightarrow \mathbf{R}$ be a continuous function such that $\left.f\right|_{M} \in C^{2}(M, \mathbf{R})$ and $\left.f\right|_{S} \equiv 0$. If

$$
c \geq \min \left\{c_{2}(r, l), k_{2}(r, l)\right\}
$$

is such that

$$
\left\|\nabla_{x}^{2} f\right\|=o\left(d(x, S)^{c}\right), \max \{1, \kappa(x)\}\left\|d_{x} f\right\|=o\left(d(x, S)^{c}\right)
$$

for $x \in M$, then there is an $\hat{f}: \mathbf{R}^{n} \rightarrow \mathbf{R}$ of class $C^{2}$ such that

$$
\left.J^{2}(\hat{f})\right|_{M}=\left.J^{2}(f \circ \pi)\right|_{M} .
$$

Proof. Let $F=f \circ \pi$; then the 2-jet of $\left.F\right|_{M}$ fulfils GHL. In fact, by Lemma II-2,

$$
\begin{aligned}
D_{i} D_{j} F(x)=\nabla^{2} f( & \left.P_{i}(x) P_{j}(x)\right) \\
& -d f\left(\sum_{h=1}^{p}\left\langle N_{h}(x), e_{i}\right\rangle L_{h, x} P_{j}(x)+\left\langle N_{h}(x), e_{j}\right\rangle L_{h, x} P_{i}(x)\right),
\end{aligned}
$$

which gives

$$
\left|D_{i} D_{j} F(x)\right| \leq\left\|\nabla_{x}^{2} f\right\|+2 p \kappa(x)\left\|d_{x} f\right\| .
$$

Our thesis now follows from (13) and GHL.

Remarks. (1) It is easy to see that, to have Theorem II- 1 for $C^{1}$ functions, we just need $\left\|d_{x} f\right\|=o\left(d(x, S)^{c}\right)$ with $c \geq \min \left\{c_{1}(r, l), k_{1}(r, l)\right\}$.

(2) Consider the elliptical bugle (Example I-1). If we fix $x$, the resulting ellipse has greatest curvature at $\left( \pm x^{a}, 0\right)$, with curvature $x^{a-2 b}$; it is not too hard to see that, for $(x, y, z)$ on $M$ near to $0, \kappa(x, y, z)=O\left(x^{a-2 b}\right)$. Thus

$$
\kappa(x, y, z)\|d f\| \leq x^{a-2 b}\|d f\|=o\left(|(x, y, z)|^{c}\right)
$$

if $\|d f\|=o\left(x^{c+2 b-a}\right)=o\left(x^{4 b-3 a}\right)$. Thus $f$ on $M$ extends if $\left\|\nabla^{2} f\right\|=o\left(x^{2(b-a)}\right)$ and $\|d f\|=o\left(x^{4 b-3 a}\right)$. 


\section{Extension of $C^{m}$ FUnCtions With $m>2$}

In this section we discuss the extendibility of functions with differentiability order higher than 2 in the case of orientable hypersurfaces of $\mathbf{R}^{n}$. The work we do will turn out at the end to be valid in the nonorientable case as well. The general codimensional case presents both computational and conceptual problems which we shall discuss later. So let's assume $M$ orientable of class $C^{m+1}$ at least and with dimension $n-1$. Let $N$ be a fixed unit normal vector field on $M$ (the only other one being $-N)$. $T$ and $\pi$ will be the standard orthogonal tubular neighborhood of $M$ and its projection, as in section II. If $L$ denotes the shape operator of $M$, from (4) of Lemma II-1 we have for $x \in T$

$$
\Delta_{x}=\left(I+\rho(x) L_{\pi(x)}\right)^{-1}
$$

where $\rho(x)=\langle x-\pi(x), N(\pi(x))\rangle$ is the "distance with sign" of $x$ from $M$.

Lemma III-1. If $x \in T$, then

$$
\Delta_{x}=\sum_{i=1}^{\infty}(-1)^{i} \rho(x)^{i} L_{\pi(x)}^{i} .
$$

Proof. $\Delta_{x}$ is self-adjoint and commutes with $L_{\pi(x)}$; hence there is a common basis of eigenvectors. Moreover the eigenvalues of $\Delta_{x}$ are the numbers $\frac{1}{1+\rho(x) \kappa_{j}(\pi(x))}$, where the $\kappa_{j}(\pi(x))$ are the principal curvatures of $M$ at $\pi(x)$. From (2) of section II, $\left|\rho(x) \kappa_{j}(\pi(x))\right|<1$; hence

$$
\frac{1}{1+\rho(x) \kappa_{j}(\pi(x))}=\sum_{i=1}^{\infty}(-1)^{i} \rho(x)^{i} \kappa_{j}(\pi(x))^{i}
$$

and the result follows.

We introduce some new notation. All subsets of $\overline{\mathbf{N}}$ will be considered ordered. If $m \in \mathbf{N}$, we set

$$
\mathbf{N}_{\leq m}=\{k \in \mathbf{N}: k \leq m\} .
$$

Then we shall identify a subset $\sigma$ of $\mathbf{N}$ with a strictly increasing map $\sigma: \mathbf{N}_{\leq \# \sigma} \rightarrow \mathbf{N}$ and we shall write $\sigma=\{\sigma(k) ; k=1, \ldots, \# \sigma\}$ (here \# denotes the cardinality). The family of subsets of $\mathbf{N}_{\leq s}$ with cardinality $j \in \overline{\mathbf{N}}$ will be denoted by $\mathcal{S}_{j, s}$. We employ the same notation with $\overline{\mathbf{N}}$ in place of $\mathbf{N}$.

We define $C^{m}(T, \pi)=\left\{F \in C^{m}(T, \mathbf{R}) ; F \circ \pi=F\right\}$. For $r \in \mathbf{N}_{\leq m}$ and $I=$ $\left(i_{1}, \ldots, i_{r}\right) \in\left(\mathbf{N}_{\leq n}\right)^{r}$, we want to estimate the values the operator $D_{I}=D_{i_{r}} \ldots D_{i_{1}}$ takes on functions in $C^{m}(T, \pi)$.

We now consider the smooth vector fields $\left\{P_{i}\right\}_{i=1, \ldots, n}$ and $N$ on $T$ defined by parallelism in $\mathbf{R}^{n}$ as

$$
P_{i}(x)=P_{i}(\pi(x)) \text { and } N(x)=N(\pi(x)) .
$$

We have $P_{i}(x) \perp N(x)$ for any $x \in T$ and $i$. Hence the $P_{i}(x)$ 's are tangents to level hypersurfaces $\{\rho=\rho(x)\}$, and $d_{x} \pi N(x)=0, d_{x} \pi P_{i}(x)=\Delta_{x} P_{i}(\pi(x))$ by Lemma II-1. So there are smooth curves $\alpha:(-\epsilon, \epsilon) \rightarrow T$ fitting $P_{i}(x)$ such that $\rho(\alpha(t)) \equiv \rho(x)$. We call such a curve $\alpha$ a standard fitting curve for $P_{i}(x)$, and we remark that the curve $\pi \circ \alpha$ fits $\Delta_{x} P_{i}(\pi(x))$. Finally, the curve $\beta:(-\epsilon, \epsilon) \rightarrow T$, $\beta(t)=t N(x)+x$, will be called the standard fitting curve for $N(x)$; we have $\pi \beta(t) \equiv \pi(x)$ and $\rho(\beta(t))=t+\rho(x)$. 
As in Section II, we have for $i \in \mathbf{N}_{\leq n}$ and $x \in T$

$$
e_{i}=P_{i, x}+\left\langle N(x), e_{i}\right\rangle N(x) .
$$

We set $\nu_{i}(x)=\left\langle N(x), e_{i}\right\rangle$ and $N_{i}(x)=\nu_{i}(x) N(x)$. Evidently, $\nu_{i} \in C^{m}(T, \pi)$ for any $i$. Using this notation and (2), the following can be easily checked:

Lemma III-2. On $T$ the identity

$$
D_{I}=\sum_{j=0}^{r} \sum_{\sigma \in \mathcal{S}_{j, s}} P_{i_{r}} \ldots P_{i_{\sigma(j)+1}} N_{i_{\sigma(j)}} P_{i_{\sigma(j)-1}} \ldots P_{i_{\sigma(1)+1}} N_{i_{\sigma(1)}} P_{i_{\sigma(1)-1}} \ldots P_{i_{1}}
$$

holds.

By the product rule for derivatives we get

Lemma III-3. The operator $D_{I}$ is a finite sum of terms of the form

$$
\nu_{j_{k}} \ldots \nu_{j_{h+1}} Q_{h}\left(\nu_{j_{h}}\right) \ldots Q_{1}\left(\nu_{j_{1}}\right) Q_{0}
$$

where the $Q_{j}$ are expressed as

$$
Q_{j}=N^{d_{s}} P_{i_{s}} N^{d_{s-1}} \ldots N^{d_{1}} P_{i_{1}} N^{d_{0}}
$$

with $s, d_{q} \in \overline{\mathbf{N}}, s+\sum_{q=o}^{s} d_{q}=r_{j}$ and $\sum_{j=0}^{h} r_{j}=r$.

From now on we shall denote the iterated composition of indexed operators by $\odot$. So we may set, dropping $j$,

$$
Q=Q_{j}=\left(\bigodot_{q=1}^{s} N^{d_{q}} P_{i_{q}}\right) N^{d_{0}}
$$

with $r=r_{j}$. Of course, some of the $d_{q}$ will be 0 . Lemma III-3 tells us we just need to estimate the image by $Q$ of functions in $C^{m}(T, \pi)$.

Lemma III-4. If $F \in C^{m}(T, \pi)$, then $N F=0$.

Proof. Use the standard fitting curve $\beta$ for $N(x)$.

Thus we shall assume from now on that $d_{0}=0$ and $s>0$ in $Q$.

The next lemma gives an expression for $Q F$ as a convergent series of functions on $T$.

Lemma III-5. Let $F \in C^{m}(T, \pi)$ and $Q=\bigodot_{q=1}^{s} N^{d_{q}} P_{i_{q}}$ with $r-s=\sum_{q=1}^{s} d_{q}=$ d. For $\ell=\left(\ell_{1}, \ldots, \ell_{s}\right) \in(\overline{\mathbf{N}})^{s}$ with $|\ell|=\ell_{1}+\cdots+\ell_{s} \geq d$ we define the following function in $C^{m}(M, \mathbf{R})$ : if $x \in M$, let

$$
L^{\ell} F(x)=\bigodot_{q=1}^{s} L^{\ell_{q}} P_{i_{q}}\left(\left.F\right|_{M}\right)(x) .
$$

Then, for $x \in T$,

$$
Q F(x)=\sum_{k=d}^{\infty}(-1)^{k} \rho(x)^{k-d} \sum_{|\ell|=k} p_{\ell} L^{\ell} F(\pi(x)),
$$

where the $p_{\ell}$ are positive integer constants which are equal to 1 if $d=0$.

Proof. Let $A=\left\{q \in \mathbf{N}_{\leq s}: d_{q} \neq 0\right\}$ and $p=\# A$. We do induction on $p$. 
Case $p=0$. In this case $d=0, Q=\bigodot_{q=1}^{s} P_{i_{q}}$ and (4) has the form

$$
Q F(x)=\sum_{k=0}^{\infty} \sum_{|\ell|=k}(-1)^{k} \rho(x)^{k} L^{\ell} F(\pi(x)) .
$$

Now, by Lemma III-1 and the Cauchy product rule, the right side of (5) is equal to

$$
\bigodot_{q=1}^{s} \Delta_{x} P_{i_{q}}\left(\left.F\right|_{M}\right)(\pi(x)) \text {. }
$$

We now use induction on $s$. If $s=1,(5)$ is just (1) of Lemma III-1. Let us assume that $s>1$ and that (5) holds for $s-1$. Then, by the remark above we get

$$
\bigodot_{q=1}^{s-1} P_{i_{q}}(F)(x)=\bigodot_{q=1}^{s-1} \Delta_{x} P_{i_{q}}\left(\left.F\right|_{M}\right)(\pi(x))
$$

on $T$.

Let $x \in T$ and let $\alpha$ be a standard fitting curve for $P_{i_{s}}(x)$. Since $\rho(\alpha(t)) \equiv \rho(x)$, $\Delta_{\alpha(t)}$ depends only on $\pi \circ \alpha$ and

$$
\begin{aligned}
Q F(x) & =\left.\frac{d}{d t}\left(\bigodot_{q=1}^{s-1} \Delta_{\alpha(t)} P_{i_{q}}\left(\left.F\right|_{M}\right)(\pi \circ \alpha(t))\right)\right|_{t=0} \\
& =\bigodot_{q=1}^{s} \Delta_{x} P_{i_{q}}\left(\left.F\right|_{M}\right)(\pi(x)),
\end{aligned}
$$

that is, formula (5).

Case $p>0$. Let us assume that (4) holds true for $p-1$ and let us order $A=\left\{q_{1}<\right.$ $\left.\cdots<q_{p}\right\}$. After setting $d_{q_{j}}=d_{j}$, we get

$$
Q=P_{i_{s}} \ldots P_{i_{q_{p}}+1} N^{d_{p}} P_{i_{q_{p}}} \ldots N^{d_{1}} P_{i_{q_{1}}} \ldots P_{i_{1}} .
$$

with $q_{1} \geq 1$.

Let

$$
Q^{\prime}=P_{i_{q_{p}}} \ldots P_{i_{q_{p-1}}+1} N^{d_{p-1}} \ldots N^{d_{1}} P_{i_{q_{1}}} \ldots P_{i_{1}} .
$$

Then, by our inductive hypothesis, there are positive integers $p_{\ell}^{\prime}$ such that

$$
Q F(x)=\sum_{k=d^{\prime}}^{\infty}(-1)^{k} \rho(x)^{k-d^{\prime}} \sum_{|\ell|=k} p_{\ell}^{\prime} L^{\ell} F(\pi(x))
$$

for $x \in T$ and $d^{\prime}=d-d_{p}$.

If $x \in T$ and $\beta$ is the standard fitting curve for $N_{x}$, we have

$$
\begin{aligned}
N Q^{\prime} F(x) & =\left.\frac{d}{d t}\left(\sum_{k=d^{\prime}}^{\infty}(-1)^{k}(\rho(x)+t)^{k-d^{\prime}} \sum_{|\ell|=k} p_{\ell}^{\prime} L^{\ell} F(\pi(x))\right)\right|_{t=0} \\
& =\sum_{k=d^{\prime}+1}^{\infty}(-1)^{k}\left(k-d^{\prime}\right) \rho(x)^{k-d^{\prime}-1} \sum_{|\ell|=k} p_{\ell}^{\prime} L^{\ell} F(\pi(x))
\end{aligned}
$$


since we can differentiate a power series term-by-term inside its radius of convergence. Applying $N_{x}$ to $Q^{\prime} d_{p}$ times, we get from (7)

$$
N^{d_{p}} Q^{\prime} F(x)=\sum_{k=d}^{\infty}(-1)^{k} \frac{\left(k-d^{\prime}\right) !}{(k-d) !} \rho(x)^{k-d} \sum_{|\ell|=k} p_{\ell}^{\prime} L^{\ell} F(\pi(x)) .
$$

Now, if $s=q_{p}$, we get our result by setting $p_{\ell}=\frac{\left(k-d^{\prime}\right) !}{(k-d) !} p_{\ell}^{\prime}$.

If $s>q_{p}$, by means of standard fitting curves of $P_{i_{s}}, \ldots, P_{i_{q_{p}}+1}$, we obtain

$$
Q F(x)=\Delta_{x} P_{i_{s}} \ldots \Delta_{x} P_{i_{q_{p}}+1} N^{d_{m}} Q^{\prime} F(x) .
$$

Thus formula (4) follows from (9), Lemma III-1 and the Cauchy product rule by setting

$$
p_{\ell}=\frac{\left(|\ell|-\ell_{s}-\cdots-\ell_{q_{p}}-d^{\prime}\right) !}{\left(|\ell|-\ell_{s}-\cdots-\ell_{q_{p}}-d\right) !} p_{\ell}^{\prime}
$$

We shall now estimate $\left|D_{I} F\right|$ on $M$ in terms of the covariant derivative norms $\left\|\nabla^{j} f\right\|$.

$\mathcal{T}_{j, s}$ will denote the set of couples $(\sigma, \tau)$, where $\sigma \in \mathcal{S}_{j, s}$ with $\sigma(1)=1$ and $\tau$ is a partition $\left\{\tau_{k}: k=1, \ldots, j\right\}$ of $\mathbf{N}_{<s} \backslash \sigma$ into $j$ subsets (allowing some of them to be empty) such that, if $\tau_{k} \neq \emptyset, \tau_{k}(\bar{h})>\sigma(k)$ for $h=1, \ldots, \# \tau_{k}$. If $X_{1}, \ldots, X_{s}$ are smooth vector fields on $M$ and $(\sigma, \tau) \in \mathcal{T}_{j, s}$, then $\nabla_{(\sigma, \tau)}\left(X_{1}, \ldots, X_{s}\right)$ will be the $j$-tuple of vector fields whose $k$-th element is

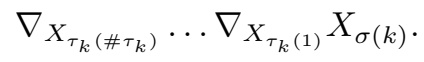

Then we may prove, by induction on $s$,

Lemma III-6. If $f \in C^{s}(M, \mathbf{R})$ and $X_{1}, \ldots, X_{s}$ are smooth vector fields on $M$,

$$
\bigodot_{q=1}^{s} X_{q} f=\sum_{j=1}^{s} \sum_{(\sigma, \tau) \in \mathcal{T}_{j, s}} \nabla^{j} f\left(\nabla_{(\sigma, \tau)}\left(X_{1}, \ldots, X_{s}\right)\right) .
$$

Given $s \in \mathbf{N}, \ell \in(\overline{\mathbf{N}})^{s}$ and $I \in\left(\mathbf{N}_{\leq n}\right)^{s}$, we define

$$
\nabla_{I, \ell}=\nabla_{L^{\ell_{s} P_{i_{s}}}} \ldots \nabla_{L^{\ell_{2} P_{i_{2}}}} L^{\ell_{1}} P_{i_{1}} .
$$

$\nabla_{I, \ell}$ is a smooth vector field on $M$ for any $I, \ell$; the number ord $\nabla_{I, \ell}=s+|\ell|-1$ will be called the order of $\nabla_{I, \ell}$. Given $q \in \overline{\mathbf{N}}$, let

$$
\hat{\chi}_{q}(x)=\sup \left\{\left\|\nabla_{I, \ell}(x)\right\|: \operatorname{ord} \nabla_{I, \ell}=q\right\}
$$

for $x \in M$.

Definition III-1. If $q \in \overline{\mathbf{N}}$, the function

$$
\chi_{q}(x)=\sup \left\{1, \hat{\chi}_{q_{1}} \cdots \cdots \hat{\chi}_{q_{k}}: q_{1}+\cdots+q_{k}=q\right\}
$$

will be called the $q$-weight of $M$ (with respect to the canonical basis).

We are now ready to enounce our estimation theorem: 
Theorem III-1. Let $r \in \mathbf{N}_{\leq m}$ and $I=\left(i_{1}, \ldots, i_{r}\right) \in\left(\mathbf{N}_{\leq n}\right)^{r}$. Let $f \in C^{m}(M, \mathbf{R})$ and $F=f \circ \pi$. Then

$$
\left|D_{I} F\right| \leq \sum_{j=1}^{r} c_{j} \chi_{r-j}\left\|\nabla^{j} f\right\|
$$

on $M$, where the $c_{j}$ are positive integer constants depending only on $r$ and $n$.

Proof. By Lemma III-3, we just need to estimate $|Q F|$ and $\left|Q \nu_{j}\right|$ on $M$, where $j \in \mathbf{N}_{\leq n}$ and $Q=N^{d} \bigodot_{q=1}^{s} P_{i_{q}}$ with $s+d \leq r$.

Now $\rho \equiv 0$ on $M$; hence formula (4) of Lemma III- 5 applied to $\nu_{j}$ and $F=f \circ \pi$ yields

$$
Q \nu_{j}(x)=(-1)^{d} \sum_{|\ell|=d} p_{\ell} L^{\ell_{s}} P_{i_{s}} \ldots L^{\ell_{1}} P_{i_{1}} \nu_{j}(x)
$$

and

$$
Q F(x)=(-1)^{d} \sum_{|\ell|=d} p_{\ell} L^{\ell_{s}} P_{i_{s}} \ldots L^{\ell_{1}} P_{i_{1}} f(x) .
$$

If $I=\left(i_{1}, \ldots, i_{s}\right)$ and $\ell^{\prime}=\ell+(1, \ldots, 0), L^{\ell} \nu_{j}=\left\langle e_{j}, \nabla_{I, \ell^{\prime}}\right\rangle$. Hence, from (13),

$$
\left|Q \nu_{j}\right| \leq \sup _{|\ell|=d}\left\{p_{\ell}\right\} \chi_{d+s}
$$

and, by (14) and Lemma III-6,

$$
|Q F| \leq \sup _{|\ell|=d}\left\{p_{\ell}\right\} \sum_{j=1}^{s} \# \mathcal{T}_{j, s} \chi_{d+s-j}\left\|\nabla^{j} f\right\| .
$$

Now (12) follows directly from (15) and (16).

Now we give the following definition:

Definition III-2. A function $f \in C^{0}(V, \mathbf{R}) \cap C^{m}(M, \mathbf{R})$ vanishing at $S$ is said to be $m$-geometrically flat on $V$ of order $c \geq 0$ if

$$
\chi_{m-j}(x)\left\|\nabla_{x}^{j} f\right\|=o\left(\delta(x, S)^{c}\right)
$$

for $j=1, \ldots, m$.

Now, by means of the GHL and Theorem III-1, we can prove an extension theorem as in Section II.

Theorem III-2. Let $f \in C^{0}(V, \mathbf{R}) \cap C^{m}(M, \mathbf{R})$. Suppose that $V$ is $(r, l)$-regular and that $f$ is m-geometrically flat of order $\min \left\{c_{m}(r, l), k_{m}(r, l)\right\}$. Then there is an $\hat{f} \in C^{m}\left(\mathbf{R}^{n}\right)$ such that $\left.J^{m}(\hat{f})\right|_{M}=\left.J^{m}(f \circ \pi)\right|_{M}$.

Remarks. (1) Obviously, we may define the $q$-weights with respect to any basis $\mathcal{B}$ of $\mathbf{R}^{n}$. Then, if $\chi_{q}^{\prime}$ is the $q$-weight defined with respect to $\mathcal{B}$, there are positive constants $A$ and $B$ such that $A \chi_{q} \leq \chi_{q}^{\prime} \leq B \chi_{q}$. This shows that the notion of $m$-geometric flatness of a given order is invariant for affine transformations of $\mathbf{R}^{n}$.

(2) If we take $-N$ instead of $N$ as unit normal field on $M$, the $q$-weights do not change. So we may work locally and extend our result to non-orientable hypersurfaces.

(3) In codimension $\geq 2$ we need, in order to define the weights, a global trivialization of the normal bundle, which does not exist in general. Moreover the weights defined in this way will depend on the chosen trivialization. In the case $m=2$, 
the use of principal curvatures gives a result which is actually weaker than Theorem III-3, but which is not tied to a choice of a (local) trivialization of the normal bundle of $M$.

\section{REFERENCES}

[B] K. Bekka, Thèse: Sur les propriétés topologiques et métriques des espaces stratifiés, Univ. de Paris-Sud, 1988.

[BT] K. Bekka, D. Trotman, Propriétés métriques de familles $\Phi$-radiales de sous-variétés différentiables, C. R. Acad. Sci. Paris Sér. I Math. 305 (1987), 389-392. MR 88m:58007

[Bi] E. Bierstone, Extension of Whitney fields from subanalytic sets, Inv. Math. 46 (1978), 277-300. MR 58:1228

[BiMP] E. Bierstone, P. Milman, W. Pawlucki, Composite differentiable functions, Duke Math. Jour. 83 (1996), 607-620. CMP 96:13

$[\mathrm{H}] \quad$ M. Hestenes', Extension of the range of a differentiable function, Duke Math. Jour. 8 (1941), 183-192. MR 2:219c

[Hi] H. Hironaka, Subanalytic sets, Number theory: in honor of Y. Akizuki, Kinokunija, Tokyo, 1973, pp. 453-483. MR 51:13275

[KO] K. Kurdyka, P. Orro, Distance géodésique sur un sous-analytique, Proceedings of the RAAG Conference Segovia 1995, Revista matematica de la Universidad Complutense de Madrid 10, num. suplementaire (1997).

[KP] K. Kurdyka, W. Pawlucki, Subanalytic version of Whitney's extension theorem, Studia Math. 124 (1997), 269-280. CMP 97:14

$[\mathrm{M}] \quad$ B. Malgrange, Ideals of differentiable functions, Oxford Univ. Press, Oxford, 1966. MR 35:3446

[Mi] J. Milnor, Morse theory, Princeton Univ. Press, Princeton, 1963. MR 29:634

[S] R. Seeley, Extension of $C^{\infty}$ functions defined in a half space, Proc. Amer. Math. Soc. 15 (1964), 625-626. MR 29:2676

[St] J. Stasica, The Whitney condition for subanalytic sets, Zeszyty Nauk. Uniw. Jag. 23 (1982), 211-221. MR 84g:32009

[T] J. C. Tougeron, Idéaux de fonctions différentiables, Springer, Berlin, 1972. MR 55:13472

[W] C. T. C. Wall, On finite $C^{k}$ left determinacy, Inv. Math. (1983), 399-405. MR 84h:58024

[Wh] H. Whitney, Analytic extension of differentiable functions defined in closed sets, Trans. Amer. Math. Soc. 36 (1934), 63-89.

[Wi] L. C. Wilson, Infinitely determined mapgerms, Canad. J. Math. 33 (1981), 671-684. MR 82k:58023

Dipartimento di Matematica, Università di Pisa, 56127 Pisa, Italy

E-mail address: ferraro@dm.unipi.it

Department of Mathematics, University of Hawail, Honolulu, Hawail 96822

E-mail address: les@math.hawaii.edu 